\title{
JNS Current and novel practice of stereotactic radiosurgery
}

\author{
JNSPG 75th Anniversary Invited Review Article \\ Douglas Kondziolka, MD, MSc, FRCSC
}

Department of Neurosurgery and Radiation Oncology, NYU Langone Health System, New York University, New York, New York

\begin{abstract}
Stereotactic radiosurgery emerged as a neurosurgical discipline in order to utilize energy for the manipulation of brain or nerve tissue, with the goal of minimal access and safe and effective care of a spectrum of neurosurgical disorders. Perhaps no other branch of neurosurgery has been so disruptive across the entire discipline of brain tumor care, treatment of vascular disorders, and management of functional problems. Radiosurgery is mainstream, supported by thousands of peer-reviewed outcomes reports. This article reviews current practice with a focus on challenges, emerging trends, and areas of investigation.
\end{abstract}

https://thejns.org/doi/abs/10.3171/2019.2.JNS181712

KEYWORDS radiosurgery; tumor; functional; vascular malformation; metastases; stereotactic; Gamma Knife

$\mathrm{S}$ TEREOTACTIC radiosurgery has revolutionized the practice of neurological surgery and radiation oncology, and has had major implications in other specialties, such as medical oncology, neurotology, pain management, neurology, and neuroimaging. The publication record across many different disorders is large, comprehensive in scope, and long-term. ${ }^{29}$ Concerns about secondary malignant transformation have been muted by longterm safety reports..$^{34,42}$ Radiation-related injury or adverse radiation effects sometimes seen as part of the expected tissue response fortunately have been relatively infrequent. Published dose-response data have led to improvements in safe outcomes. This report describes elements of current practice, expanding concepts, and new avenues for investigation.

\section{Technical Aspects of Stereotactic Radiosurgery}

A number of different technologies are available to perform radiosurgery procedures. These continue to include photon- and proton-based devices. Established devices used to care for large numbers of patients include the Leksell Gamma Knife (Elekta AB), the CyberKnife (Accuray Inc.), and different modified linear accelerator systems. Over 1 million patients have been treated with Gamma Knife radiosurgery, and it is clear that the concept has been used in large numbers of patients with intracranial disorders and a smaller number with selected extracranial tumors and vascular malformations.

Over the last 40 years, most patients who have undergone stereotactic radiosurgery have done so after application of a rigid stereotactic frame. Several linear accelerator systems utilize mask fixation, which can provide acceptable precision and accuracy when done well. However, for small-volume targets and targets in critical locations, I continue to believe that rigid frame fixation provides the utmost in accuracy and confidence. As with any device, the frame must be applied properly and securely. We use mask fixation during multi-session radiosurgery with the Gamma Knife ICON unit in order to target larger-volume lesions or brain metastasis resection beds. I have found this fixation to be acceptable in appropriately selected patients who could tolerate the mask and who limited their body motion during irradiation. Most patients can only tolerate mask fixation for up to 30 minutes. In contrast, selected patients with multiple metastases and complex skull base tumors requiring multiple small isocenters can require radiosurgical exposures much longer than 30 minutes. In such cases, rigid frame placement is much more suitable.

Imaging sequences continue to improve. High-resolution MRI with or without contrast administration is the key to proper target identification and delineation of critical structures. Depending on the imaging system manufacturer, specific long relaxation time sequences can be ideal for cranial nerve delineation. A number of radiosurgical 
software systems can auto-segment structures of interest (i.e., Brainlab Elements software), which can be helpful to the clinician. What remains deficient is our ability to quickly study tumor metabolism. Also, we lack information on the blood flow through an arteriovenous malformation (AVM), which may be useful for dose planning. Although functional imaging can be performed prior to radiosurgery and co-registered, it is not often utilized in routine dose planning.

\section{Radiobiology}

The value of radiosurgery is dependent on the radiobiological effect on the target tissue. There are numerous ways to evaluate the potential radiobiological effect. ${ }^{22}$ This is important not only in dose planning but also in outcome assessment. Typically, a clinician considers the effects of dose to the lesion and the dose to the area surrounding the lesion. Little work has been done on the importance of the dose falloff or "dose gradient." Studies have shown that this may not be relevant in pain relief after trigeminal neuralgia radiosurgery. A recent analysis suggested that a reduced gradient may be associated with better tumor control in the care of small brain metastases.

The dose rate may be an important radiobiological factor. This can vary depending on different radiosurgical devices, but also on the activity of the cobalt-60 in a Gamma Knife surgery. Although a faster dose rate may have a stronger radiobiological effect, the actual effect of this is not clear. A computer algorithm is used to calculate the prescribed dose, but some investigators argue that a different algorithm may be better. This would utilize the concept of "biologically effective dose" on the target tissue. Work in this area is ongoing. In my opinion, future radiosurgical devices should improve on the radiobiological effect. This would allow a more potent intrinsic target effect with less irradiation of surrounding tissue. It may also be that an individual tumor should be targeted using a different dose profile to different portions of the tumor that may be more or less radiation sensitive. For example, treatment of a metastasis with central necrosis, indicative of intratumoral hypoxia and radioresistance, may be planned differently from treatment of a tumor of similar volume that is homogeneously solid.

\section{Arteriovenous Malformations}

There have been many articles published on the response of AVMs to stereotactic radiosurgery. ${ }^{6,7}$ These studies have looked at clinical outcomes, dose response, safety, lesion location, dose staging, and adverse effects. Since the publication of ARUBA (A Randomized Trial of Unruptured Brain Arteriovenous Malformations), there have been a number of articles that compare radiosurgical outcomes in patients with unruptured AVMs to data from that trial. ${ }^{11}$ The data support the use of radiosurgery for unruptured AVMs in patients expected to live at least 12-15 years. In this fashion, the value of AVM obliteration over time with potential morbidity outweighs the outcomes associated with observation alone. ${ }^{11}$

Some centers have used a multi-session radiosurgery approach to target larger-volume AVMs. There are not strong outcomes data on this concept, although I have seen some impressive outcomes presented by Wurm and colleagues (personal communication). Others have used a low initial dose to the entire volume, repeating radiosurgery as necessary several years later for persistent AVMs. Others, including our group, have used a volume-staged approach in which components of the AVM are irradiated at intervals of 4-5 months or so. Typically, the target volume at each session is less than $10 \mathrm{~cm}^{3}$, although it may be as high as $15 \mathrm{~cm}^{3}$. It is clear that we need to do better for patients with symptomatic, large AVMs that are not amenable to either resection or endovascular embolization-due to excessive surgical risk or likelihood of suboptimal response, respectively. I continue to believe that a better and more quantitative understanding of blood flow to an AVM is the key to improving the radiosurgical effect. With this information, radiosurgical energy could be directed differently to different portions of the AVM. For malformations in critical locations, we commonly use functional MRI information as part of dose planning (Fig. 1).

One key limitation of AVM radiosurgery remains the latency interval to vascular obliteration of the target. Although the data suggest that the hemorrhage rate may be slightly reduced during this latency interval, hemorrhage can still occur. We are aggressive in the use of endovascular techniques to address hemodynamic risk factors for hemorrhage. It may be that as experience with transvenous embolization techniques improves, and the morbidity profile is more clearly understood, combined approaches utilizing radiosurgery and endovascular surgery may lead to significant improvements in overall outcome.

\section{Vestibular Schwannomas}

Stereotactic radiosurgery is highly effective in the care of patients with small- and medium-sized vestibular schwannomas. ${ }^{15,37}$ It has also been evaluated in selected patients with larger tumors. ${ }^{24}$ The goal of radiosurgery is long-term inactivation of tumor growth and preservation of cranial nerve function. Although facial nerve function is preserved in over $99 \%$ of patients, preservation of hearing is less successful.

Before recommending any form of treatment, an understanding of the natural history of tumor growth is important. Unfortunately, many past studies have been conducted with low-resolution imaging and use of linear dimension measurements only-sometimes with unusual definitions of tumor growth (such as an intracanalicular tumor only growing if it becomes extracanalicular). For this reason, many patients are told of a typical slow growth pattern or that their tumor "may not grow at all." Two recent studies (from the Mayo Clinic ${ }^{23}$ and NYU [unpublished data]) used serial volumetric measurements and high-resolution imaging techniques to define tumor growth patterns. The Mayo study showed that $69 \%$ of patients had volumetric growth within a median of 1.1 years. ${ }^{23}$ The NYU study showed that over an average 2-year follow-up interval, approximately $30 \%$ of patients did not have significant tumor growth $(<20 \%$ by volume) and that in $70 \%$ of cases the tumor did grow by more than $20 \%$, with half of those tumors growing by more than $100 \%$ per year. Thus there are 

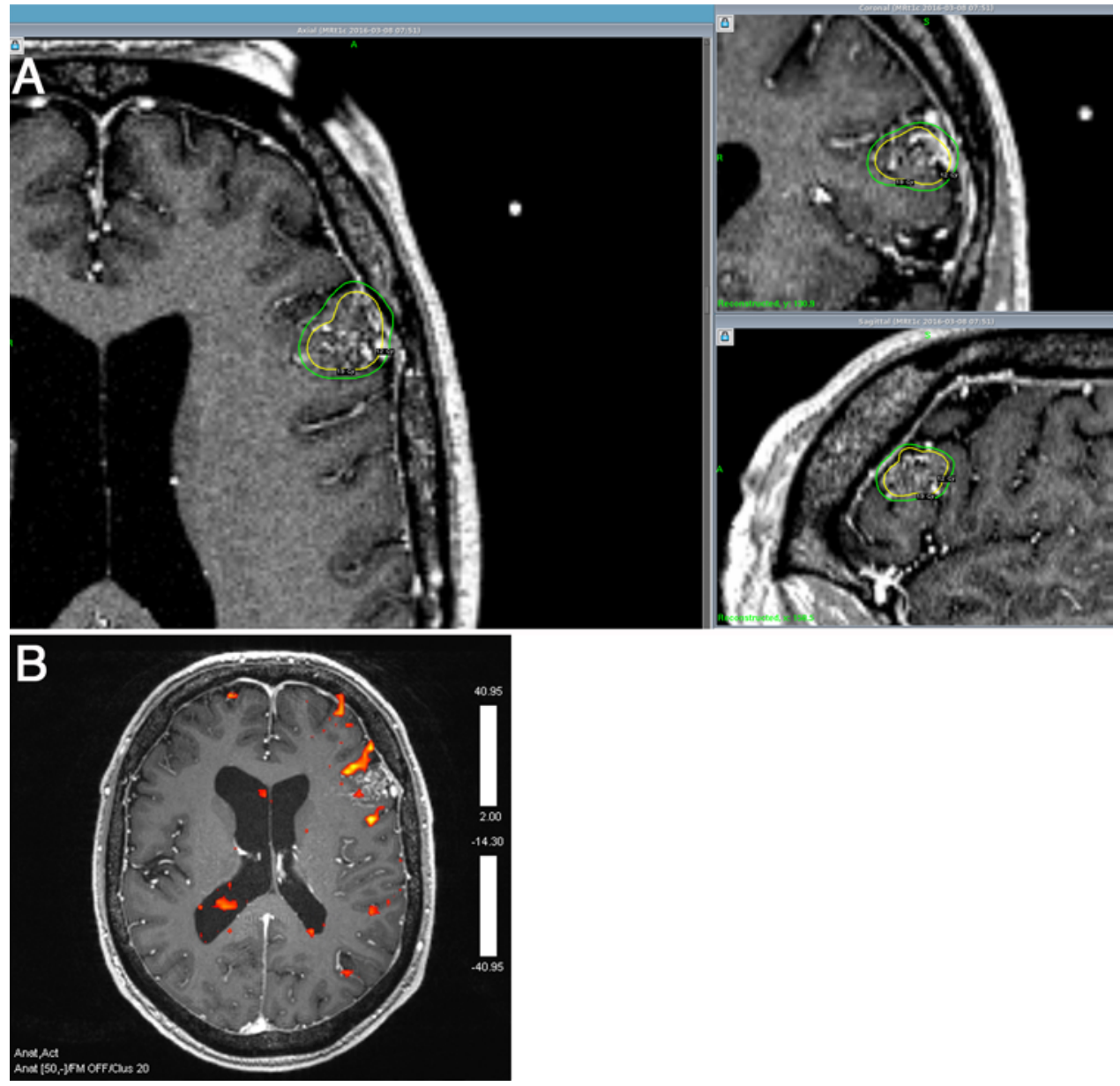

FIG. 1. Case of a 63-year-old man with a left frontal AVM close to Broca's speech area. A: Gamma Knife radiosurgery dose plan (19 Gy). B: Functional activation for word generation is shown surrounding the AVM. Two years after radiosurgery, the AVM showed reduced vascularity with no edema or new symptoms. Figure is available in color online only.

both slower and faster growth groups. The average growth rate across the entire series was $33 \%$ by volume per year.

The dose prescribed to the margin of a vestibular schwannoma typically varies at most centers between 11 and 13 Gy. Lower doses are sometimes used for patients for whom hearing preservation may be one goal, particularly to lower the mean cochlear dose. However, it is not clear that this is indicated. While several studies do show that a reduction of the mean cochlear dose below $4.2 \mathrm{~Gy}$ may be advantageous for hearing preservation, this may be too simplistic an expectation. It is likely that the quality of pre-radiosurgery hearing is most important, as well as other factors difficult to study such as the time duration for tumor growth and the vascular effect of the tumor on the cochlear nerve. This is why important data show better hearing outcomes with earlier management, when hearing is at its highest level. ${ }^{1}$ The concept of "watch and wait" for smaller tumors has been followed most commonly by tumor growth and hearing loss. ${ }^{37}$ As for the use of low radiation doses, my personal concern is that a dose reduction may lead to a higher failure rate for longer-term tumor control without clear benefit to hearing. Indeed, with precise dose planning, a dose elevation (14 Gy) may be war- ranted in some patients who already have poor hearing to increase the tumor control rate. My expectation is that this would have little adverse effect on facial function. This is an area for further research.

Over the last decade, the goal of hearing preservation has been associated with the strategies for tumor management. For larger-volume tumors this could include a partial resection followed by radiosurgery to the residual mass. A more recent concept is the use of cochlear implants in patients with an irradiated and controlled tumor who still have some low-level residual hearing integrity (Fig. 2). At our own center we have had some gratifying outcomes using this approach in a small number of patients. My expectation is that this concept will be used increasingly in patients who also have reduced hearing in the contralateral ear.

\section{Meningiomas}

Radiosurgery has revolutionized the management of intracranial meningiomas. ${ }^{21}$ Prior to its use, management consisted of observation or resection, sometimes performed numerous times for recurrent tumors. Fractionated radia- 

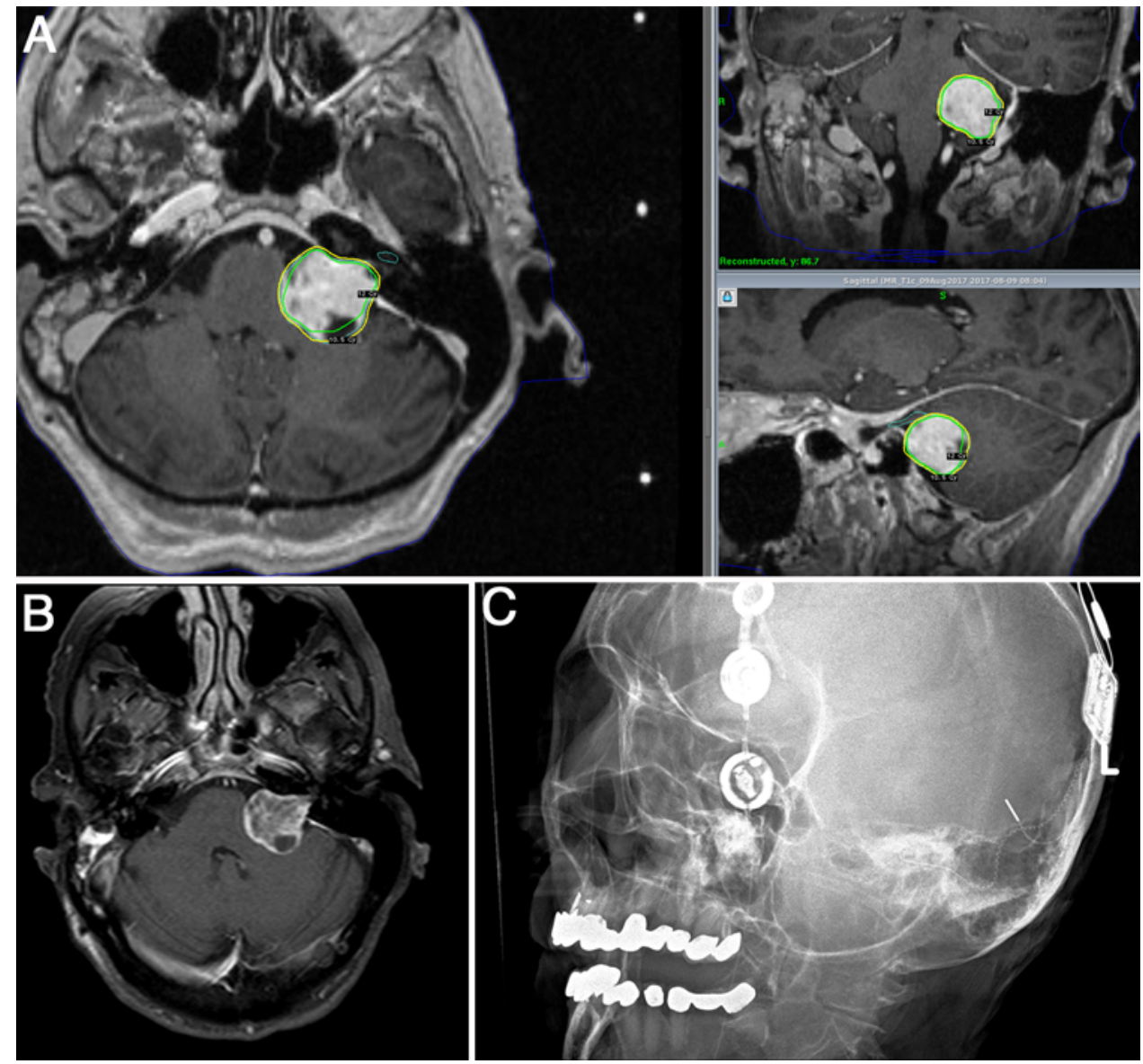

FIG. 2. Case of an 82-year-old woman with a recurrent left vestibular schwannoma receiving repeat radiosurgery (10.5 Gy). A: Radiosurgery dose plan. B: Axial post-contrast short relaxation time MR images obtained 6 months after radiosurgery showing reduced central contrast uptake. C: Radiograph showing the left cochlear implant that was placed to address hearing loss that existed before radiosurgery. The patient experienced improved hearing with the implant. Figure is available in color online only.

tion therapy had a limited role for primary management but was used for difficult, more aggressive histological types. For patients with tumors in critical locations, such as at the skull base or along the venous sinuses, radiosurgery assumed a primary role if the lesions were symptomatic and small. Long-term data show excellent tumor control rates (above 90\%) with low risk for new neurological problems. ${ }^{13,27}$ Indeed for patients treated relatively soon after diagnosis, cranial nerve deficits can often be improved.

Experience in the care of many thousands of patients with long-term published results has substantiated a durable and effective tumor margin dose between 12 and 16 Gy in most cases. Most patients with a grade I tumor receive 12-14 Gy. Grade II or III tumors should be managed with the higher doses, and indeed patients with malignant meningiomas should be considered for additional fractionated radiation therapy.

The future of meningioma radiosurgery research centers around several concerns including its use for largervolume tumors, obtaining a better understanding of cranial nerve tolerance, and evaluating how radiosurgical effects can influence peritumoral brain edema. For selected cases of larger-volume tumors in which additional surgical resection was not thought feasible, we have used a multisession radiosurgery approach. ${ }^{8}$ We are collecting data on outcomes since little has been published. The optimal dose paradigm remains to be determined. We use $4 \mathrm{~Gy} \times$ 5 sessions or 5 Gy $\times 5$ sessions depending on volume and perioptic location.

\section{Brain Metastases}

At most centers, brain metastasis represents the most common indication for stereotactic radiosurgery. Over the last 30 years, perhaps for no other brain tumor has management changed so much. Whole brain radiation therapy, once the most commonly used approach, is now rare. ${ }^{4,32}$ Resection remains important in the treatment of larger-volume symptomatic tumors associated with brain edema. ${ }^{36}$ However, radiosurgery can also be effective in patients whose symptoms can be managed with corticosteroid therapy until tumor regression is seen. Whereas radiosurgery was only used for single metastases and small numbers of tumors in the 1990s, it is now used widely for higher numbers of tumors and indeed no clear upper limit 

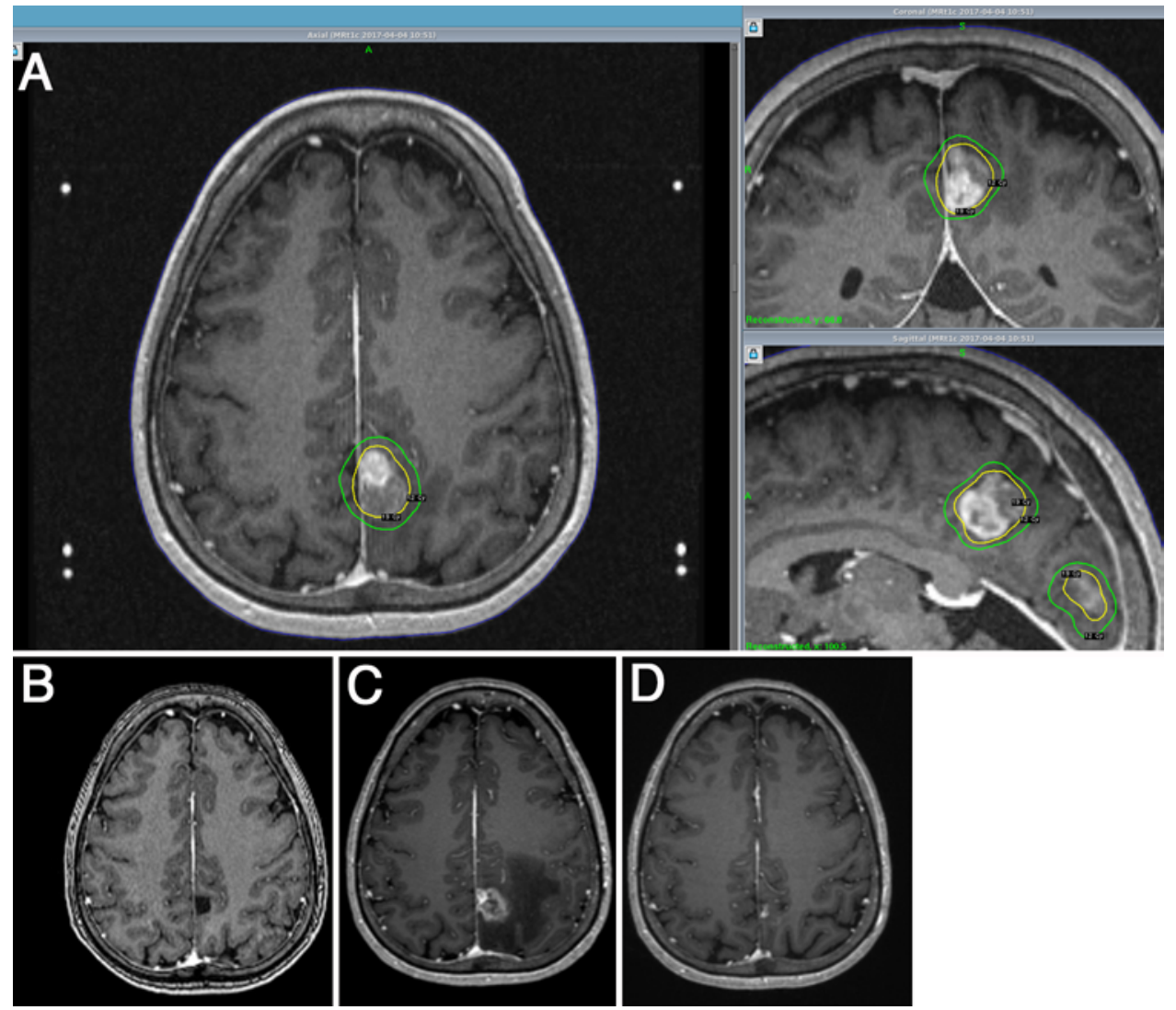

FIG. 3. Case of a 58-year-old woman with metastatic breast cancer and multiple metastases. A: The radiosurgery dose plan for the left mesial parietal tumor (18 Gy). B-D: Axial post-contrast short relaxation time MR images showing apparent resolution of the tumor 6 months after radiosurgery (B), the appearance of new enhancement and edema 16 months after radiosurgery (C), and regression of imaging changes 2 months after 2 cycles of bevacizumab (D). Figure is available in color online only.

has been set. ${ }^{47}$ We commonly perform radiosurgery in patients with 10-25 tumors. Whole brain radiation therapy for micrometastatic disease has been replaced in many patients by systemic therapies with some degree of brain tissue penetrance. Whole brain radiation therapy still remains an important approach for miliary disease and diffuse leptomeningeal disease.

Different radiosurgical technologies help clinicians manage multiple brain metastases in different ways. Some are more suited to the efficient care of higher numbers of brain tumors in cancer patients (i.e., Gamma Knife technique). Others employ a single isocentric approach to deliver multiple radiation fields and can do so quickly using some types of linear accelerator technology. Such rapid dose delivery may be at the expense of the delivery of higher doses to the surrounding remote brain, but any detrimental effects of this remain to be determined.

Importantly, clinical outcomes research should focus on specific cancer types and not include all forms of tumors just because they are metastatic. ${ }^{44}$ Since radiobiological response, systemic therapies, and clinical expectations differ, most of our studies should not include breast cancer, lung cancer, and melanoma in the same report.

An important avenue for research should be in the evaluation of short-course bevacizumab therapy in the care of patients with adverse radiation effects. ${ }^{5}$ Avoiding longer-term corticosteroid use is important in this patient population where quality of life is crucial, and avoidance of steroid myopathy is necessary so that systemic therapy can continue uninterrupted. Some of our patients have had impressive responses to just 2 doses of bevacizumab with reduction of contrast enhancement, rapid resolution of edema, and avoidance of dexamethasone treatment (Fig. 3).

\section{Trigeminal Neuralgia}

Stereotactic radiosurgery is a common indication for medically refractory trigeminal neuralgia. There have been numerous published reports over the last 25 years that detail clinical outcomes. At most centers, the technique is standard. High-resolution imaging is used to facilitate precise small-volume irradiation of a portion of the trigeminal nerve anterior to the pons. Targeting accuracy is the key to this surgery, and indeed there have been cases in which structures other than the nerve have been targeted. In the vast majority of patients, the nerve can be well seen. Identification can be more challenging in patients who have a small subarachnoid space or sometimes in those who have had prior microvascular decompression. High-resolution long relaxation time sequences are excellent for cranial 
nerve identification. The maximum dose delivered to the trigeminal nerve is usually 80 or $85 \mathrm{~Gy}$, keeping the brainstem surface at a low dose. ${ }^{43}$ In many patients, pain relief can occur rapidly over a few days or weeks, although the average time to noted benefit is approximately 1 month, and the effect continues to build over several months. For patients who are in severe unrelenting pain and are having trouble eating or drinking, other techniques, such as percutaneous rhizotomy or microvascular decompression, may have advantages for more rapid pain relief. Whether the cause of the pain is idiopathic, from vascular compression, from multiple sclerosis, or due to some unknown cause, radiosurgery offers a minimal-access strategy that is preferable to medical treatment. ${ }^{45}$ For patients with a benign basal tumor compression as the cause, we currently favor a combined dose plan that provides a high dose to the proximal trigeminal nerve of $80 \mathrm{~Gy}$, with the dose falloff shaped to envelop the tumor to a margin dose of 12-13 Gy. This concept was first reported by Kim et al. ${ }^{19}$

There have been numerous studies that compare outcomes between radiosurgery and other surgical approaches. Microvascular decompression in properly selected patients has an excellent record of clinical benefit with long-lasting relief in many patients. We think that for all younger or medically healthy patients with clear vascular compression identified on imaging, microvascular decompression should be a primary surgical choice.

A current question goes to the timing of a surgical procedure in this disorder. For the most part, patients are typically seen by surgeons years after the initial diagnosis. This is because the course of pain can wax and wane, and usually one or more medical therapies are tried. In our own practice the time to a first neurosurgical procedure had typically been about 8 years after pain onset. Lunsford and colleagues (Mousavi et al.) ${ }^{30}$ reported their data showing improved outcomes in patients who had radiosurgery within the first year of onset. This may make intuitive sense-treating patients before the physiology of trigeminal neuralgia becomes more refractory to any form of therapy. We are currently evaluating this concept within a large multicenter patient cohort.

Another question goes to the management of recurrent trigeminal neuralgia. How many radiosurgery procedures can a patient undergo? The effects of a second radiosurgery to boost the effects of the first, typically performed in patients who had a good initial response and then developed some pain recurrence, have been reported ${ }^{30}$ The data show that pain relief can certainly be achieved in many cases, but fewer patients become pain free and can stop medication use. The value of a third radiosurgery was also reported..$^{41}$ We consider this in patients with trigeminal neuralgia related to multiple sclerosis or in selected other patients who have a long trigeminal nerve course.

\section{Other Facial Pain Syndromes and Headache}

Given the value of radiosurgery to affect trigeminal nerve function and physiology, it has been used to modulate trigeminal afferent input for other craniofacial pain disorders in addition to trigeminal neuralgia. The main utility has been for chronic cluster headache $(\mathrm{CCH}) .{ }^{16} \mathrm{Ra}$ - diosurgery options included trigeminal nerve radiosurgery (similar to that performed for trigeminal neuralgia) or combined trigeminal nerve and sphenopalatine ganglion radiosurgery. The latter concept, which I prefer, is geared to affect both afferent and efferent physiology, given that $\mathrm{CCH}$ can be associated with vasomotor symptoms. Typically, radiosurgery is considered in cases of medically refractory $\mathrm{CCH}$, usually after failure of drug therapies, such as anticonvulsant agents, prednisone, calcium channel blockers, oxygen therapy, and others. Some studies show a higher incidence of facial sensory loss after radiosurgery in $\mathrm{CCH}$ patients than in trigeminal neuralgia patients (cause unclear). A multicenter study did not show a clear benefit when the 1-target and 2-target concepts were compared.

Dr. Peter Jannetta suggested the use of radiosurgery to us for sphenopalatine neuralgia, a facial pain syndrome that can be difficult to treat. The pain is typically periorbital and perinasal, and can be associated with tearing, nasal discharge, and red eye. We consider radiosurgery in patients who have had a positive, though usually shortlived, response to a local anesthetic nerve block. ${ }^{33}$ This must be performed by a clinician skilled in that procedure. The ganglion can be identified in the pterygopalatine fossa on MRI and CT. In each case we have a neuroradiologist work with us to evaluate the images for targeting. We use a single 8-mm isocenter and a maximum dose of 85-90 Gy. There are case reports of the successful use of Gamma Knife radiosurgery to the combined trigeminal nerve and sphenopalatine ganglion targets for patients with SUNCT syndrome (short-lasting unilateral neuralgiform headache with conjunctival injection and tearing). It is considered one of the "trigeminal autonomic cephalgias." 12 We have also performed radiosurgery with benefit, using CT guidance to the bilateral greater palatine nerves, in a patient with burning mouth syndrome and palatal pain.

\section{Movement Disorder and Behavioral Radiosurgery}

Brain lesioning is highly effective for well-selected patients with tremor or obsessive-compulsive disorders, and it has been evaluated in selected patients with other disorders (i.e., dystonia, thalamic pain). Radiosurgical thalamotomy (targeting the ventralis intermedius nucleus) is well reported for essential tremor, Parkinson's disease tremor, and multiple sclerosis-associated tremor. ${ }^{31}$ The outcome can be gratifying. As with any radiosurgical effect, the response is delayed in onset. One concern is the potential development of a hyper-response leading to a symptomatic adverse radiation effect (causing hemiparesis and/or hemisensory loss). I am particularly concerned about patients who have diabetes mellitus and may not tolerate the vascular radiation effect-or corticosteroid therapy should it later be necessary. Recently, another energy-based lesioning technique, high-frequency focused ultrasound, has been used for thalamic lesioning. Outcomes for tremor are also good and, importantly, immediate. However, the morbidity profile remains a concern. ${ }^{3}$ Typically, lesioning is considered in patients not well suited to deep brain stimulation.

Stereotactic radiosurgery for the treatment of medically refractory, severe obsessive-compulsive disorder can 

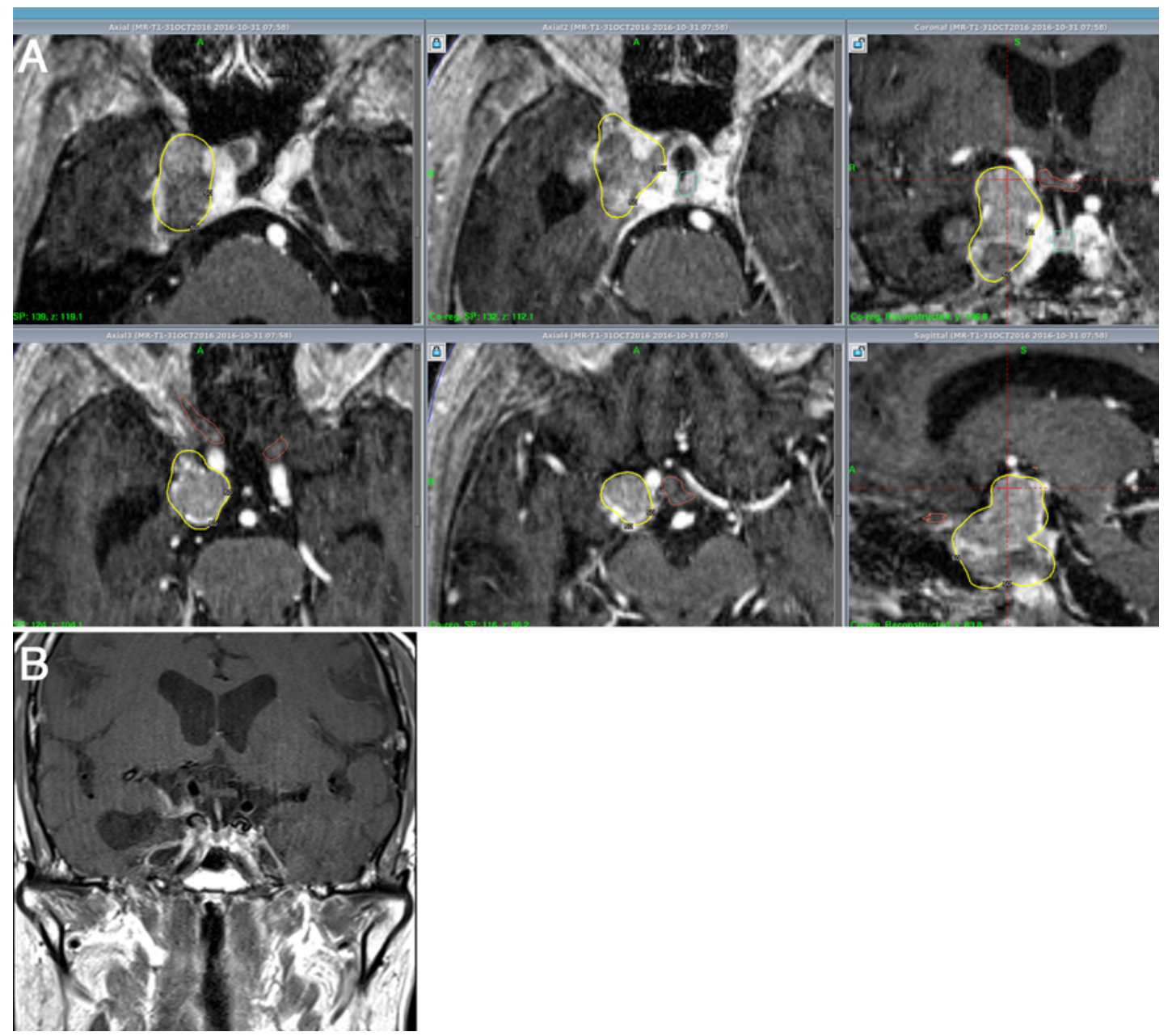

FIG. 4. Case of a 71-year-old with a recurrent prolactinoma in the right cavernous sinus. A: Radiosurgery dose plan for 4 Gy at each of 5 sessions using the Gamma Knife ICON unit. B: Coronal post-contrast short relaxation time MR image obtained 2 years after radiosurgery showing marked tumor regression. Figure is available in color online only.

be highly effective in this challenging patient population. ${ }^{20}$ Bilateral anterior capsulotomy, targeted inferiorly toward the nucleus accumbens, is performed. A common maximum radiosurgery dose is $140-150 \mathrm{~Gy}$, although others have used lower or higher doses. ${ }^{28}$ One- or two-isocenter planning is used. A blinded randomized trial was performed and showed benefit to radiosurgery over control. ${ }^{28}$ Nevertheless, this remains an infrequent and underutilized procedure.

\section{Pituitary Adenomas}

Radiosurgery has an established role for non-secretory or secretory pituitary adenomas, particularly after prior resection. ${ }^{39}$ The majority of patients with non-secreting tumors who undergo radiosurgery have residual or recurrent tumor involving the cavernous sinus. The tumor control rate after radiosurgery is high, and typically the dose necessary to achieve such a response is relatively low (12-14 Gy). Patient selection is dependent upon the proximity of the tumor to the optic nerves and chiasm. With high-reso- lution imaging and precise dose planning, tumors can be targeted right up to the optic system, although sometimes a small dose reduction at that point is needed. We aim to keep the optic system at a maximum point dose below 10 Gy. For tumors more intimate to or compressive of optic structures, and for which additional resection may not be feasible, a multi-session radiosurgery approach can be beneficial. We use a strategy of 4-5 Gy at each of 5 sessions using mask fixation (Fig. 4).

One current controversy is the timing of radiosurgery after resection. If residual tumor remains, should it be observed or should radiosurgery be performed electively several months later? A recent report notes that earlier radiosurgery provides a better tumoral response and a lower rate of late pituitary insufficiency. ${ }^{35}$ Thus, if clear tumor remains on imaging, we favor early radiosurgery particularly when the tumor is maximally away from the optic nerve and chiasm. If there is no clear tumor visible and residual tumor is only suspected, then serial imaging is advocated to identify any later growth should it occur.

Growth hormone-, cortisol-, or prolactin-secreting tu- 
mors constitute the majority of secretory adenomas managed with radiosurgery. ${ }^{10,26}$ These can be either macro- or microadenomas. Typically, we stop any antihormonal therapy for at least 4 weeks before SRS, which we think improves tumor radiosensitivity. Higher doses are used as guided by optic safety. Most current studies show an approximate $25 \%$ rate of some degree of pituitary insufficiency over the decade after treatment.

\section{Glomus Jugulare Tumors}

Glomus tumors respond to radiation. When they are identified relatively early and when their volume is not excessive, stereotactic radiosurgery is an effective primary management approach. Embolization and partial resection is certainly of value for large tumors and can be used to more rapidly reduce symptoms such as disabling tinnitus. However, the risk of cranial nerve dysfunction can be high, depending on tumor anatomy. For that reason, radiosurgery is a relatively low-risk approach that leads to long-term tumor control in a high percentage of patients. ${ }^{40}$ Fractionated radiation therapy can also be effective, but radiosurgery has the benefits of precise, regional tissue sparing and a stronger radiobiological intratumoral effect, and it can be done in a single session. Over the last decade, we have been performing radiosurgery on increasing numbers of patients with glomus tumors. Not infrequently, patients will have bilateral tumors.

\section{Glial Tumors}

The role of radiosurgery has evolved over time. For patients with small-volume residual or recurrent pilocytic astrocytomas, clinical outcomes data show excellent tumor response rates. ${ }^{17,18}$ Most tumors are in critical brain locations (and otherwise would have been resected completely). For recurrent ependymomas or anaplastic ependymomas, radiosurgery is similarly effective. However, the propensity for the latter type to recur in other subarachnoid locations is high.

Over the last 30 years, the utility of radiosurgery for patients with glioblastoma multiforme has changed. Prior to the use of drug therapies, radiosurgery was a frequently used boost to standard external beam radiotherapy. It is clear that radiation is effective, and the concept was to give as much as possible and safely. Then during the 1990s, as the utility of one drug after another was tried, radiosurgery was used less often, typically for smaller-volume local recurrences in patients in whom almost everything else had failed. In some patients, a local control benefit could be achieved. But for the most part, radiosurgery was an afterthought. Interestingly, virtually all published reports showed similar outcomes. Radiosurgery was associated with a local control and survival benefit in patients with smaller-volume recurrent tumors, when matched to similar patients who were not treated with this modality. However, its role as initial up-front management before radiotherapy was not supported.

Current concepts consider the role of radiotherapy together with checkpoint inhibition therapies, and to target areas of white matter tumoral invasion (the FLAIR change seen on MRI).

\section{Spinal Radiosurgery}

It is hard to imagine that when the radiosurgery concept was being developed as a tool for brain surgery, spinal applications were in any way discussed. There were too many obstacles, the most important of which were the lack of reasonable imaging tools to define disease states and regional neural structures (other than plain radiographs) and the lack of target immobilization. But when these obstacles were addressed in some way by the early to mid1990s, applications for human neuro-oncology (and to a very limited extent, spinal vascular malformations) began in earnest. Twenty-five years later, high-resolution imaging, different immobilization techniques, motion management, imaging-based tracking, separation surgery, and published clinical outcomes for benign and malignant tumors established spinal radiosurgery as an important primary therapeutic approach for many patients. ${ }^{14,25,38,46}$ Different commercially available systems, mainly using linear accelerator-based devices, became widely available. The impressive results using single-session radiosurgery in the brain, with its unique radiobiological features, were extended extracranially-first to the spine, and then to selected other organ systems. Even conventional fractionated radiotherapy, when coupled with the stereotactic, image-guided principles of neurosurgery, evolved into the concept of stereotactic body radiation therapy (SBRT).

Current and future research continues to provide outcome data for patients with benign and malignant tumors, evaluate the tolerance of regional normal tissues, clarify the role of radiosurgery with resection or systemic therapies, study reasons for failure, evaluate repeat radiosurgery, study effects on pain and quality of life, determine longer-term effects on bony integrity, and assess the durability of the response. JNS: Spine has published many such reports.

\section{Imaging Tools for Response Assessments}

Evaluating the radiosurgery response can be easy when the tumor regresses in size or the AVM no longer is identified on an angiogram. However, for those patients who have more contrast uptake or more edema after radiosurgery, the imaging interpretation can be challenging. Is the enhancement nodular or ill defined? Is it just inflammatory with blood-brain barrier disruption from radiation vasculopathy, or does it represent actual tumor nodularity? Were the same imaging sequences available for comparison both before and after radiosurgery? If a fluorodeoxyglucose (FDG) PET scan or perfusion MRI shows some degree of metabolic change, how does one know that that appearance is new? Why is methionine PET used in many European centers but not in the United States?

First, we think that the use of a comprehensive set of MRI sequences before radiosurgery and at each followup visit assists the clinicians and radiologists at image interpretation. Second, standardizing the use of other techniques (PET, perfusion imaging, permeability imaging, spectroscopy, 7-T CEST [chemical exchange saturation transfer] metabolite imaging, and others) is important for research efforts where enough data can be collected to be meaningful. 


\section{Adverse Radiation Effects}

As noted, it can be difficult to distinguish radiationrelated effects from tumor recurrence. This challenge is similar to the identification of recurrence versus scar after resection or blood-brain barrier changes and enhancement from drug effects after systemic therapies. When an adverse radiation effect is identified, particularly when symptomatic, standard options have included simple observation, use of oral corticosteroids, or resection if mandated. The use of other agents such as vitamin E or pentoxifylline has been tested. Hyperbaric oxygen therapy has not proven to be of reliable value. The use of bevacizumab, targeting vascular endothelial growth factor, often at lower doses and for a small number of administrations, has proven to be an extremely valuable therapy that can lead to fairly rapid improvement in edema, reduction in contrast uptake, and improvement in symptoms. ${ }^{2,5,9}$ Concerns remain related to possible side effects. It is also relatively expensive. A number of studies have reported its utility in the management of adverse radiation effects, and we think it is an underutilized approach. In contrast, long-term corticosteroid therapy is often associated with metabolic changes, proximal myopathy, and other unwarranted effects. The value of bevacizumab as treatment for selected brain tumors has proven somewhat disappointing. However, its greatest role may be in the treatment of adverse radiation effects, where a primary component of the effect is radiation vasculopathy. Future research and published outcomes will hopefully substantiate the role of short-course bevacizumab, bringing it into mainstream care and making it available for all patients, and significantly reducing our reliance on corticosteroids.

\section{References}

1. Akpinar B, Mousavi SH, McDowell MM, Niranjan A, Faraji $\mathrm{AH}$, Flickinger JC, et al: Early radiosurgery improves hearing preservation in vestibular schwannoma patients with normal hearing at the time of diagnosis. Int J Radiat Oncol Biol Phys 95:729-734, 2016

2. Aslan A, Kaya ZB, Bulduk EB, Ocal O, Ucar M, Erpolat OP, et al: Prophylactic bevacizumab may mitigate radiation injury: an experimental study. World Neurosurg 116:e791-e800, 2018

3. Boutet A, Ranjan M, Zhong J, Germann J, Xu D, Schwartz ML, et al: Focused ultrasound thalamotomy location determines clinical benefits in patients with essential tremor. Brain 141:3405-3414, 2018

4. Brown PD, Jaeckle K, Ballman KV, Farace E, Cerhan JH, Anderson SK, et al: Effect of radiosurgery alone vs radiosurgery with whole brain radiation therapy on cognitive function in patients with 1 to 3 brain metastases: a randomized clinical trial. JAMA 316:401-409, 2016

5. Chung C, Bryant A, Brown PD: Interventions for the treatment of brain radionecrosis after radiotherapy or radiosurgery. Cochrane Database Syst Rev 7:CD011492, 2018

6. Cohen-Inbar O, Starke RM, Lee CC, Kano H, Huang P, Kondziolka D, et al: Stereotactic radiosurgery for brainstem arteriovenous malformations: a multicenter study. Neurosurgery 81:910-920, 2017

7. Cohen-Inbar O, Starke RM, Paisan G, Kano H, Huang PP, Rodriguez-Mercado R, et al: Early versus late arteriovenous malformation responders after stereotactic radiosurgery: an international multicenter study. J Neurosurg 127:503-511, 2017
8. Combs SE, Ganswindt U, Foote RL, Kondziolka D, Tonn JC: State-of-the-art treatment alternatives for base of skull meningiomas: complementing and controversial indications for neurosurgery, stereotactic and robotic based radiosurgery or modern fractionated radiation techniques. Radiat Oncol 7:226, 2012

9. Deibert CP, Ahluwalia MS, Sheehan JP, Link MJ, Hasegawa $\mathrm{T}$, Yomo $\mathrm{S}$, et al: Bevacizumab for refractory adverse radiation effects after stereotactic radiosurgery. J Neurooncol 115:217-223, 2013

10. Ding D, Mehta GU, Patibandla MR, Lee CC, Liscak R, Kano $\mathrm{H}$, et al: Stereotactic radiosurgery for acromegaly: an international multicenter retrospective cohort study. Neurosurgery 84:717-725, 2019

11. Ding D, Starke RM, Kano H, Mathieu D, Huang P, Kondziolka D, et al: Radiosurgery for cerebral arteriovenous malformations in A Randomized Trial of Unruptured Brain Arteriovenous Malformations (ARUBA)-eligible patients: a multicenter study. Stroke 47:342-349, 2016

12. Effendi K, Jarjoura S, Mathieu D: SUNCT syndrome successfully treated by gamma knife radiosurgery: case report. Cephalalgia 31:870-873, 2011

13. Harrison G, Kano H, Lunsford LD, Flickinger JC, Kondziolka D: Quantitative tumor volumetric responses after Gamma Knife radiosurgery for meningiomas. J Neurosurg 124:146154, 2016

14. Gerszten PC, Goldschmidt E, Gerszten K: Determining radiographic outcome after spine radiosurgery-not as straightforward as one might think. Spine J 16:299-301, 2016

15. Golfinos JG, Hill TC, Rokosh R, Choudhry O, Shinseki M, Mansouri A, et al: A matched cohort comparison of clinical outcomes following microsurgical resection or stereotactic radiosurgery for patients with small- and medium-sized vestibular schwannomas. J Neurosurg 125:1472-1482, 2016

16. Kano H, Kondziolka D, Mathieu D, Stafford SL, Flannery TJ, Niranjan A, et al: Stereotactic radiosurgery for intractable cluster headache: an initial report from the North American Gamma Knife Consortium. J Neurosurg 114:1736-1743, 2011

17. Kano H, Kondziolka D, Niranjan A, Flickinger JC, Lunsford LD: Stereotactic radiosurgery for pilocytic astrocytomas part 1: outcomes in adult patients. J Neurooncol 95:211-218, 2009

18. Kano H, Niranjan A, Kondziolka D, Flickinger JC, Pollack IF, Jakacki RI, et al: Stereotactic radiosurgery for pilocytic astrocytomas part 2: outcomes in pediatric patients. J Neurooncol 95:219-229, 2009

19. Kim SK, Kim DG, Se YB, Kim JW, Kim YH, Chung HT, et al: Gamma Knife surgery for tumor-related trigeminal neuralgia: targeting both the tumor and the trigeminal root exit zone in a single session. J Neurosurg 125:838-844, 2016

20. Kondziolka D, Flickinger JC, Hudak R: Results following gamma knife radiosurgical anterior capsulotomies for obsessive compulsive disorder. Neurosurgery 68:28-33, 2011

21. Kondziolka D, Patel AD, Kano H, Flickinger JC, Lunsford LD: Long-term outcomes after gamma knife radiosurgery for meningiomas. Am J Clin Oncol 39:453-457, 2016

22. Kondziolka D, Shin SM, Brunswick A, Kim I, Silverman JS: The biology of radiosurgery and its clinical applications for brain tumors. Neuro Oncol 17:29-44, 2015

23. Lees KA, Tombers NM, Link MJ, Driscoll CL, Neff BA, Van Gompel JJ, et al: Natural history of sporadic vestibular schwannoma: a volumetric study of tumor growth. Otolaryngol Head Neck Surg 159:535-542, 2018

24. Lefranc M, Da Roz LM, Balossier A, Thomassin JM, Roche $\mathrm{PH}$, Regis J: Place of Gamma Knife stereotactic radiosurgery in grade 4 vestibular schwannoma based on case series of 86 patients with long-term follow-up. World Neurosurg 114:e1192-e1198, 2018 
25. Ling DC, Flickinger JC, Burton SA, Heron DE, Quinn AE, Bejjani GK, et al: Long-term outcomes after stereotactic radiosurgery for spine metastases: radiation dose-response for late toxicity. Int J Radiat Oncol Biol Phys 101:602-609, 2018

26. Mehta GU, Ding D, Patibandla MR, Kano H, Sisterson N, Su YH, et al: Stereotactic radiosurgery for Cushing's Disease: results of an international multicenter study. J Clin Endocrinol Metab 102:4284-4291, 2017

27. Mehta GU, Zenonos G, Patibandla MR, Lin CJ, Wolf A, Grills I, et al: Outcomes of stereotactic radiosurgery for foramen magnum meningiomas: an international multicenter study. J Neurosurg 129:383-389, 2018

28. Miguel EC, Lopes AC, McLaughlin NCR, Norén G, Gentil $\mathrm{AF}$, Hamani C, et al: Evolution of gamma knife capsulotomy for intractable obsessive-compulsive disorder. Mol Psychiatry 24:218-240, 2019

29. Mitrasinovic S, Zhang M, Appelboom G, Sussman E, Moore JM, Hancock SL, et al: Milestones in stereotactic radiosurgery for the central nervous system. J Clin Neurosci 59:1219, 2019

30. Mousavi SH, Niranjan A, Huang MJ, Laghari FJ, Shin SS, Mindlin JL, et al: Early radiosurgery provides superior pain relief for trigeminal neuralgia patients. Neurology 85:21592165,2015

31. Niranjan A, Raju SS, Kooshkabadi A, Monaco E III, Flickinger JC, Lunsford LD: Stereotactic radiosurgery for essential tremor: retrospective analysis of a 19-year experience. Mov Disord 32:769-777, 2017

32. Patel KR, Burri SH, Boselli D, Symanowski JT, Asher AL, Sumrall A, et al: Comparing pre-operative stereotactic radiosurgery (SRS) to post-operative whole brain radiation therapy (WBRT) for resectable brain metastases: a multi-institutional analysis. J Neurooncol 131:611-618, 2017

33. Pollock BE, Kondziolka D: Stereotactic radiosurgical treatment of sphenopalatine neuralgia. Case report. J Neurosurg 87:450-453, 1997

34. Pollock BE, Link MJ, Stafford SL, Parney IF, Garces YI, Foote RL: The risk of radiation-induced tumors or malignant transformation after single-fraction intracranial radiosurgery: results based on a 25-year experience. Int J Radiat Oncol Biol Phys 97:919-923, 2017

35. Pomeraniec IJ, Kano H, Xu Z, Nguyen B, Siddiqui ZA, Silva D, et al: Early versus late Gamma Knife radiosurgery following transsphenoidal surgery for nonfunctioning pituitary macroadenomas: a multicenter matched-cohort study. J Neurosurg 129:648-657, 2018

36. Prabhu RS, Miller KR, Asher AL, Heinzerling JH, Moeller BJ, Lankford SP, et al: Preoperative stereotactic radiosurgery before planned resection of brain metastases: updated analysis of efficacy and toxicity of a novel treatment paradigm. J Neurosurg [epub ahead of print December 14, 2018. DOI: 10.3171/2018.7.JNS181293]

37. Régis J, Carron R, Park MC, Soumare O, Delsanti C, Thomassin JM, et al: Wait-and-see strategy compared with proactive Gamma Knife surgery in patients with intracanalicular vestibular schwannomas: clinical article. J Neurosurg 119 Suppl:105-111, 2013
38. Robin AM, Yamada Y, McLaughlin LA, Schmitt A, Higginson D, Lis E, et al: Stereotactic radiosurgery: the revolutionary advance in the treatment of spine metastases. Neurosurgery 64 (CN Suppl 1):59-65, 2017

39. Sheehan JP, Starke RM, Mathieu D, Young B, Sneed PK, Chiang VL, et al: Gamma Knife radiosurgery for the management of nonfunctioning pituitary adenomas: a multicenter study. J Neurosurg 119:446-456, 2013

40. Sheehan JP, Tanaka S, Link MJ, Pollock BE, Kondziolka $\mathrm{D}$, Mathieu D, et al: Gamma Knife surgery for the management of glomus tumors: a multicenter study. J Neurosurg 117:246-254, 2012

41. Tempel ZJ, Chivukula S, Monaco EA III, Bowden G, Kano H, Niranjan A, et al: The results of a third Gamma Knife procedure for recurrent trigeminal neuralgia. J Neurosurg 122:169-179, 2015

42. Wolf A, Naylor K, Tam M, Habibi A, Novotny J, Liščák R, et al: Risk of radiation-associated intracranial malignancy after stereotactic radiosurgery: a retrospective, multicentre, cohort study. Lancet Oncol 20:159-164, 2019

43. Wolf A, Tyburczy A, Ye JC, Fatterpekar G, Silverman JS, Kondziolka D: The relationship of dose to nerve volume in predicting pain recurrence after stereotactic radiosurgery in trigeminal neuralgia. J Neurosurg 128:891-896, 2018

44. Wolf A, Zia S, Verma R, Pavlick A, Wilson M, Golfinos JG, et al: Impact on overall survival of the combination of BRAF inhibitors and stereotactic radiosurgery in patients with melanoma brain metastases. J Neurooncol 127:607-615, 2016

45. Xu Z, Mathieu D, Heroux F, Abbassy M, Barnett G, Mohammadi AM, et al: Stereotactic radiosurgery for trigeminal neuralgia in patients with multiple sclerosis: a multicenter study. Neurosurgery 84:499-505, 2019

46. Yamada Y, Katsoulakis E, Laufer I, Lovelock M, Barzilai O, McLaughlin LAZ, et al: The impact of histology and delivered dose on local control of spinal metastases treated with stereotactic radiosurgery. Neurosurg Focus 42(1):E6, 2017

47. Yamamoto M, Serizawa T, Shuto T, Akabane A, Higuchi Y, Kawagishi J, et al: Stereotactic radiosurgery for patients with multiple brain metastases (JLGK0901): a multi-institutional prospective observational study. Lancet Oncol 15:387-395, 2014

\section{Disclosures}

The author reports a financial relationship with Brainlab, involving research not directly related to this article.

\section{Correspondence}

Douglas Kondziolka: NYU Langone Health System, New York University, New York, NY. douglas.kondziolka@nyulangone.org. 